\title{
Coherent $x$-ray imaging of defects in colloidal crystals
}

\author{
J. Gulden, ${ }^{1}$ O. M. Yefanov, ${ }^{1}$ A. P. Mancuso, ${ }^{1}$ V. V. Abramova, ${ }^{2}$ J. Hilhorst,${ }^{2}$ D. Byelov, ${ }^{2}$ I. Snigireva, ${ }^{3}$ A. Snigirev, ${ }^{3}$ \\ A. V. Petukhov, ${ }^{2}$ and I. A. Vartanyants ${ }^{1, *}$ \\ ${ }^{1}$ Deutsches Electronen-Synchrotron (DESY), Notkestraße 85, D-22607 Hamburg, Germany \\ ${ }^{2}$ Van't Hoff Laboratory for Physical and Colloid Chemistry, Debye Institute, University of Utrecht, Padualaan 8 , \\ 3508 TB Utrecht, The Netherlands \\ ${ }^{3}$ ESRF, 6 rue Jules Horowitz, F-38043 Grenoble, France
}

(Received 1 March 2010; revised manuscript received 20 May 2010; published 10 June 2010)

\begin{abstract}
Coherent $\mathrm{x}$-ray diffractive imaging (CXDI) was applied to reveal the structure of colloidal crystals. The colloidal sample was illuminated by a coherent x-ray beam through a $7 \mu \mathrm{m}$ pinhole aperture. The resulting diffraction patterns contain several Bragg peaks and an additional interference structure between the peaks due to the coherent illumination of a finite part of the sample. The inversion of these diffraction patterns reveals the arrangement of colloidal particles in a face-centered cubic (fcc) lattice as well as defects in the form of stacking faults in the (111) planes.
\end{abstract}

DOI: 10.1103/PhysRevB.81.224105

PACS number(s): 61.05.C-, 42.30.Rx, 61.72.Nn, 82.70.Dd

\section{INTRODUCTION}

Real crystalline materials, in contrast to an idealized picture of perfect crystals, contain a broad spectrum of defects. These defects determine most of the mechanical, optical and electronic properties of the crystals. ${ }^{1}$ Different methods are used to reveal these defects and their distribution in the material either directly, or indirectly. X-rays are a very attractive probe due to their high penetration depth and are routinely used for investigating defects in different materials. For example, $x$-ray topography ${ }^{2}$ was one of the first imaging $x$-ray methods to visualize defects in crystals. Using this approach defects are imaged indirectly by the induced strain fields. Presently, well developed tomographic methods can reliably reveal the three-dimensional (3D) distribution of defects in materials, ${ }^{3}$ but the resolution is typically limited to a few microns. Unfortunately, most of the present x-ray methods fail to visualize the defect core down to the atomic level in a bulk material. Here we propose to use coherent $\mathrm{x}$-ray diffractive imaging (CXDI) to map such defects in colloidal crystals.

Self-organized colloidal crystals can be used as the basis for novel functional materials such as photonic crystals, which may find applications in future solar cells, LEDs, lasers or even as the basis for circuits in optical computing and communication. For these applications crystal quality is crucial and monitoring the defect structure of real colloidal crystals is essential. ${ }^{4}$

CXDI is based on the idea that if a finite object is illuminated with coherent radiation and its corresponding far-field diffraction pattern is measured and sampled sufficiently, then this diffraction pattern can be inverted uniquely ${ }^{5}$ using iterative phase retrieval methods. ${ }^{6-8}$ After its first experimental demonstration ${ }^{9}$ CXDI was applied to the imaging of different samples and materials (see for reviews Refs. 10 and 11). This method was also successfully implemented for imaging crystalline materials ${ }^{12-14}$ by a local fine scan of the reciprocal space in the vicinity of a chosen Bragg peak from the sample. By inverting this 3D distribution of the diffracted intensity, the shape and strain field inside a crystalline par- ticle can be obtained. ${ }^{13}$ However, local measurements around a selected Bragg peak can reveal only a continuous distribution of electron density and strain inside a crystalline sample. ${ }^{15}$ We demonstrate here that if several Bragg peaks of a coherently illuminated finite crystalline sample are measured and inverted simultaneously, the resulting image will contain a rich amount of information about the position of the local scatterers, including possible defects.

In practice, the experimental realization of these ideas is not so straightforward, even with short wavelength $\mathrm{x}$ rays. It is desirable to measure several Bragg peaks simultaneously on one detector for a fixed position of the sample. With typical atomic distances in crystalline materials this will require energies in the range of $100 \mathrm{keV} .{ }^{16}$ High energy electron beams can be also used to image the structure of nanocrystals with the coherent imaging technique ${ }^{17,18}$ providing measurements of several Bragg peaks on a detector. However, as for most electron scattering methods, this approach is limited to small samples in the range of few tens of nanometers. For $\mathrm{x}$-ray scattering methods one possible approach is to use large-period structures. ${ }^{19}$ This is conveniently provided by nature in the form of colloidal crystals with a period in the range of a few hundred nanometers. Illuminating such samples with hard $\mathrm{x}$-rays produces a diffraction pattern with many Bragg peaks mapped simultaneously on a twodimensional (2D) detector ${ }^{20}$ and provides a convenient way to study the arrangement of colloidal particles.

\section{EXPERIMENT}

In our present coherent imaging experiment we used a thin film of a colloidal crystalline sample on a glass substrate. It was grown by the convective assembly technique ${ }^{21,22}$ using polystyrene microspheres (diameter 425 $\mathrm{nm}$, relative standard deviation $<5 \%$ ). The spheres were synthesized by emulsifier-free emulsion polymerization of styrene using potassium persulphate as an initiator. ${ }^{23}$ Glass microslides were immersed in an aqueous suspension of microspheres with a volume fraction of about $0.5 \%$. The temperature of the film growth was $50{ }^{\circ} \mathrm{C}$. The grown crystal- 

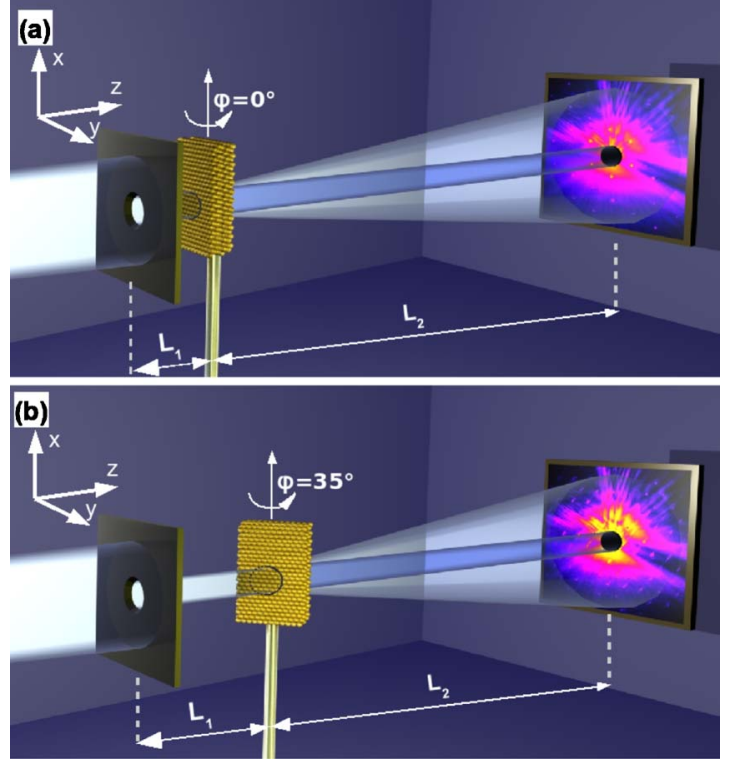

FIG. 1. (Color online) Schematic view of the CXDI experiment showing the pinhole, the sample and the detector. (a) Measurements at azimuthal angle $\varphi=0^{\circ}$ (b) $\varphi=35^{\circ}$.

line films have a face-centered cubic (fcc) structure ${ }^{24}$ and were typically 20 to 30 layers thick.

The experiment was performed at the micro-optics test bench at the ID06 beamline of the European Synchrotron Radiation Facility (ESRF) with an incident x-ray energy of $14 \mathrm{keV}$. The geometry of our experiment (see Fig. 1) allows for rotation of the sample around the vertical axis perpendicular to the incident x-ray direction. A $6.9 \mu \mathrm{m}$ pinhole was positioned at a distance $L_{1}$ in front of the colloidal crystal. The pinhole selects a highly coherent part of the beam and produces a finite illumination area. It was produced by laser drilling in a $250 \mu \mathrm{m}$ thick Pt foil and finally etched by a focused ion beam (FIB). The final shape has a tapered profile with a minimum thickness of $7 \mu \mathrm{m}$. The initial orientation of the sample (with azimuthal angle $\varphi=0^{\circ}$ ) corresponds to the direction of the incident $\mathrm{x}$-rays along the [111] direction of the fcc colloidal crystal and was perpendicular to the surface normal of the sample [Fig. 1(a)]. The pinhole to sample distance was $L_{1}=1 \mathrm{~mm}$ in this case. The sample was scanned in the $x, y$ directions perpendicular to the incident beam with the possibility to locally map large-period structures at different positions of the sample. Rotating the sample around the $x$ axis allows the measurement of different sets of diffraction planes. Particularly important was the direction of the incident $\mathrm{x}$-rays along the [110] direction of the colloidal sample lattice at $\varphi=35^{\circ}$. At this position the set of (111) planes was aligned along the incident beam. In this geometry [see Fig. 1(b)] the pinhole sample distance was increased to $L_{1}=25 \mathrm{~mm}$, due to geometrical constraints.

The diffraction data were recorded using a Photonic Science 12 bit charge-coupled device (CCD) with 4005 $\times 2671$ pixels each $9 \times 9 \mu \mathrm{m}^{2}$ in size. The detector was placed at the distance $L_{2}=3.96 \mathrm{~m}$ behind the sample with a corresponding resolution of $\Delta q=0.16 \mu \mathrm{m}^{-1}$ per pixel. Due to saturation of the detector at the position of the Bragg peaks, short (10 s) and long (30 s) time exposures were used.
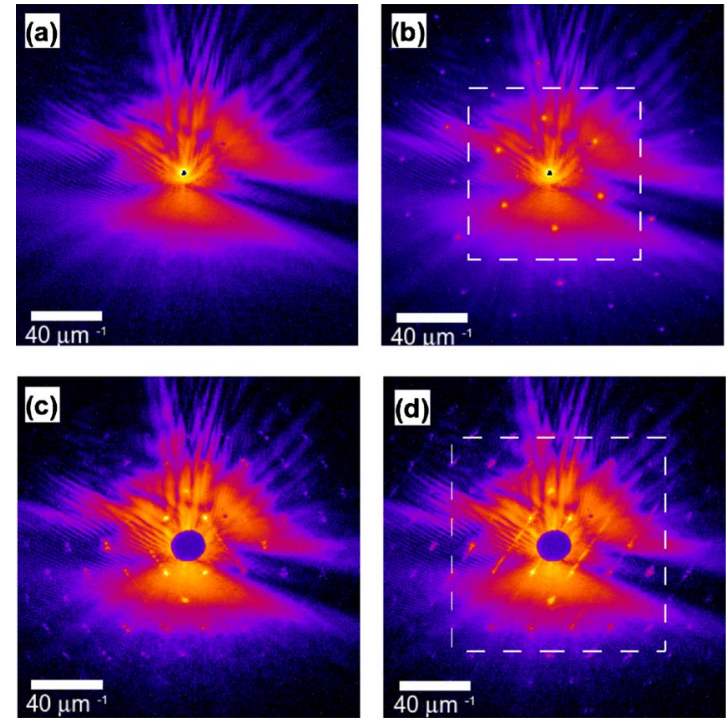

FIG. 2. (Color online) Measured diffraction patterns. (a) Diffraction pattern from the pinhole alone. [(b)-(d)] Diffraction patterns from the pinhole and the sample at different azimuthal angle orientations (b) $\varphi=0,[(\mathrm{c})$ and (d) $] \varphi=35^{\circ}$. Two sets of streaks originating at Bragg peaks in (c) and (d) are indications of stacking fault defects present in the colloidal sample. Marked regions in (b) and (d) correspond to the area used for the reconstruction. All diffraction patterns are shown on a logarithmic scale.

Each measurement was repeated 10 times to enhance the signal to noise ratio.

\section{RESULTS AND DISCUSSION}

\section{A. Measurements at azimuthal angle $\varphi=0^{\circ}$}

A diffraction pattern from the pinhole without the sample is shown in Fig. 2(a). Strong fringes, typical of an Airy pattern $^{25}$ from a circular aperture, are clearly seen in this diffraction pattern. Despite cleaning the aperture with a FIB to minimize the roughness of the aperture, an unavoidable diffuse background (due to imperfections) contaminates the diffraction data (see Fig. 2). Positioning the sample just after the aperture yields a similar set of fringes as in the previous case, centered at $q=0$ [Fig. 2(b)]. In addition, due to the long range order in the colloidal crystal, several orders of Bragg peaks are easily visible in the diffraction patterns. The strongest are the hexagonal set of 220 Bragg peaks typical for scattering from a fcc structure. Each of these Bragg peaks contains a few orders of diffraction fringes similar to those at $q=0$, due to the finite aperture in front of the sample. This is similar to observations in our previous experiments on coherent scattering from large-period finite structures. ${ }^{19} \mathrm{In}$ addition to the allowed 220 Bragg peaks, we also observed much weaker forbidden peaks [1/3(224) in our case]. Their appearance is an indication of defects in the crystal.

The measured diffraction data were inverted by applying the guided hybrid input-output GHIO algorithm. ${ }^{26}$ Starting with eight parallel runs with random initial phases, five generations were used, with 1000 iterations of HIO (Ref. 6) and one iteration of the error-reduction (ER) algorithm at the end 

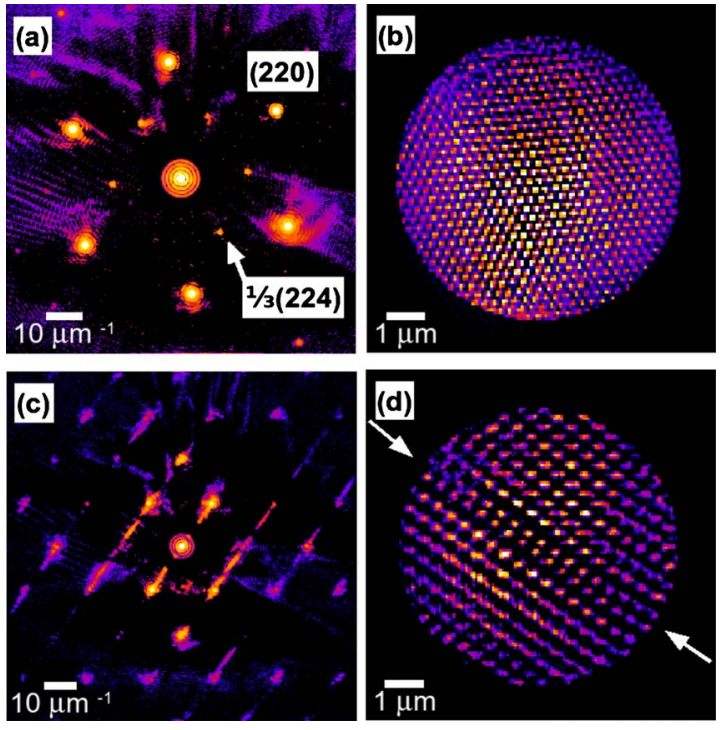

FIG. 3. (Color online) [(a) and (c)] Difference diffraction patterns (in logarithmic scale) obtained as a result of the subtraction of the scaled diffraction pattern of the pinhole from the measured diffraction patterns of the sample (a) $\varphi=0$, (c) $\varphi=35^{\circ}$. Black regions correspond to negative values and were left to evolve freely in the reconstruction. The central part around $q=0$ was implemented from the result of the reconstruction after 20 iterations. [(b) and (d)] Reconstruction of the colloidal sample from the diffraction patterns (a) $\varphi=0$, (c) $\varphi=35^{\circ}$. The arrows in (d) point to the defect in the crystal.

of each generation. In all reconstructions the positivity constraint was enforced.

The first attempts to reconstruct the sample from the measured diffraction pattern [Fig. 2(b)] have shown that the internal structure of the colloidal sample was strongly suppressed by the high level of the varying background. We attribute this to the presence of a strong incoherent background in the diffraction pattern in Fig. 2(a), originating from the pinhole. To improve the quality of the reconstruction we subtracted a scaled diffraction pattern of the pinhole [Fig. 2(a)] from the diffraction pattern of the sample [Fig. 2(b)]. We explored the effect of subtracting the incoherent background of the pinhole from the diffraction patterns of the sample with varying scale factors. We found that a scaling factor of 1.3 was optimal to remove artifacts from the reconstructed images. After this procedure the 220 Bragg peaks, and especially the fringes around them, are clearly distinguished on a reduced background [see Fig. 3(a)]. Negative values, shown in black in the difference diffraction pattern in Fig. 3(a), were left to evolve freely in the reconstruction procedure. However, reconstructions performed from these data sets were not stable due to the large region of missing data, especially in the low $q$ region. ${ }^{27}$ To stabilize the reconstruction process we kept the central region (with $q<5.44 \mu \mathrm{m}^{-1}$ ) of the reconstructed diffraction pattern fixed after 20 initial iterations [see Fig. 3(a)]. We then proceed with these fixed low $q$ values and as a result the following reconstructions were stable.

Implementing all these procedures, we performed a reconstruction of the diffraction pattern shown in Fig. 3(a) and
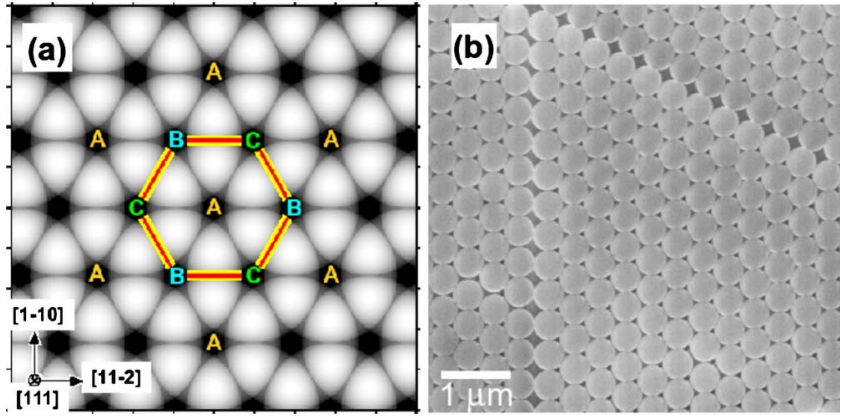

FIG. 4. (Color online) (a) Schematic projection of three (111) planes in a perfect fcc crystal. The presence of A, B, and C positions in a single image reduces the apparent periodicity of the crystal. (b) SEM image of the crystal surface of a similar colloidal crystal in the presence of the stacking fault. The resulting structure resembles the reconstructed image in Fig. 3(d).

obtained a real space image of a colloidal sample presented in Fig. 3(b). This image represents a projection of the "atomic" structure of the colloidal crystal along the [111] direction. The hexagonal structure is clear across the whole illuminated region, with only slightly lower intensity values of the image around the edges of the pinhole aperture. As a consequence of the image being a projection of 3D arrangement of colloidal particles, the periodicity does not correspond to the colloidal interparticle distance $d$ in a single crystalline layer. This is illustrated in Fig. 4(a), which is a schematic drawing of three hexagonal (111) layers projected onto a flat image. In this projection all three possible positions (A, B and C) of a hexagonal layer in an fcc crystal contribute to the signal, resulting in a reduced periodicity of $d / \sqrt{3}$, as indicated by the hexagon.

The major differences from the results of previous work with CXDI on crystalline samples ${ }^{13}$ are clearly demonstrated in Fig. 3(b). Instead of a continuous shape and strain field reconstructed from the measurements of diffraction patterns around a single Bragg peak, the hexagonal structure shown in Fig. 3(b) gives the projected positions of the colloidal particles.

This can be understood by the following arguments. ${ }^{15,28}$ An electron density, $\rho(\mathbf{r})$, of the illuminated part of the colloidal sample can be written as

$$
\rho(\mathbf{r})=S(\mathbf{r})\left[s(\mathbf{r}) \otimes \rho_{\infty}(\mathbf{r})\right],
$$

where $S(\mathbf{r})$ is the shape function of the illuminated area defined in our experiment by the pinhole in the front of the sample, $s(\mathbf{r})$ is the projection of the electron density of one colloidal particle, $\rho_{\infty}(\mathbf{r})=\Sigma_{n} \delta\left(\mathbf{r}-\mathbf{r}_{\mathbf{n}}\right)$ is a periodic function defining the $2 \mathrm{D}$ lattice, $\otimes$ is a convolution sign, and $\mathbf{r}$ is a $2 \mathrm{D}$ coordinate vector in the plane of the sample surface. Considering that the scattering amplitude $A(\mathbf{q})$ in the kinematical approximation is just the Fourier transform (FT) of a periodic electron density of colloidal crystal, after simple transformation $^{28}$ it can be presented in the following form:

$$
A(\mathbf{q})=\sum_{n} F\left(\mathbf{h}_{\mathbf{n}}\right) S\left(\mathbf{q}-\mathbf{h}_{\mathbf{n}}\right) .
$$

Here $F\left(\mathbf{h}_{\mathbf{n}}\right)$ is the form factor of the electron density of the colloidal particle sampled at Bragg positions $\mathbf{h}_{\mathbf{n}}=\mathbf{2} \pi \mathbf{H}_{\mathbf{n}}$ de- 
fined by the $2 \mathrm{D}$ reciprocal lattice vectors $\mathbf{H}_{\mathbf{n}}$ and $S(\mathbf{q})$ is the FT of the pinhole function $S(\mathbf{r})$. For a close packed system, such as colloidal crystals, the Bragg positions are separated by a considerable distance in reciprocal space. This means that the form factor $F\left(\mathbf{h}_{\mathbf{n}}\right)$ is sampled by only a few points in reciprocal space and modulates the values of the Bragg peaks. ${ }^{24}$ In our specific case, we limited the area of reciprocal space for reconstruction by including only the strongest 220 Bragg peaks which have a similar intensity. ${ }^{42}$ The form factor $F\left(\mathbf{h}_{220}\right)$ at the position of the 220 Bragg peaks can be taken out of the sum in the scattering amplitude in Eq. (2) giving

$$
A(\mathbf{q})=F\left(\mathbf{h}_{220}\right) \sum_{n} S\left(\mathbf{q}-\mathbf{h}_{\mathbf{n}}\right) .
$$

Inversion of this expression can provide the $2 \mathrm{D}$ projection of the positions of the colloidal particles and the shape function of the illuminated region $S(\mathbf{r})$ encoded in the Bragg peak positions $\mathbf{h}_{\mathbf{n}}$ and in the function $S(\mathbf{q})$. However, information about the shape of individual colloidal particles [encoded in the form-factor $F\left(\mathbf{h}_{\mathbf{n}}\right)$ ] cannot be retrieved. In our specific case it is just a scaling factor in the expression for the amplitude. Following this analysis we attribute the finite size of the particles in the reconstruction to a finite resolution and not to the reconstruction of the colloidal particle shape function. The smearing of individual particle positions can be also attributed to a small shift in the positions of the colloidal particles in the projected lattice (there are about 20-30 layers in the $z$ direction). We estimate the resolution by performing line scans through the reconstructed image in Fig. 3(b) and measuring the widths of the peaks by Gaussian fit. In this case we observe a full width half maximum (FWHM) of 95 $\mathrm{nm}$ at the particle positions in Fig. 3(b).

\section{B. Measurements at azimuthal angle $\varphi=35^{\circ}$}

The diffraction patterns measured at an angle of $\varphi=35^{\circ}$ [see Figs. 2(c) and 2(d)] were especially intriguing. They show strong streaks of varying intensity originating at the Bragg peaks with an angle of $-55^{\circ}$ [Fig. 2(c)] or $+55^{\circ}$ [Fig. 2(d)] to the horizontal direction-depending on the specific location of the sample. It is well known from previous studies $^{24}$ of similar colloidal systems that such streaks in reciprocal space are induced by stacking faults in the fcc structure in the (111) planes.

We reconstructed these diffraction patterns as described earlier and the result of this reconstruction [from the data in Fig. 3(c)] is presented in Fig. 3(d). The "atomicity" of the colloidal crystal sample is again present in the reconstruction. In addition, a stacking fault [indicated by arrows in Fig. 3(d)] appears as a break in the "correct" $\mathrm{ABC}$ ordering. ${ }^{29} \mathrm{We}$ note that colloidal self-assembled crystals are known to be prone to stacking disorder. Often a random hexagonal close packed (rhcp) structure is observed, ${ }^{30-35}$ which consists of a random mixture of fcc and hcp planes. Also, the stacking direction is often random so that one finds $\mathrm{ABC}$ - as well as ACB-type fcc sequences. The reconstructed image in Fig. 3(d) reveals a different situation. One can see a stacking fault, which consists of two hep planes, and two fcc domains
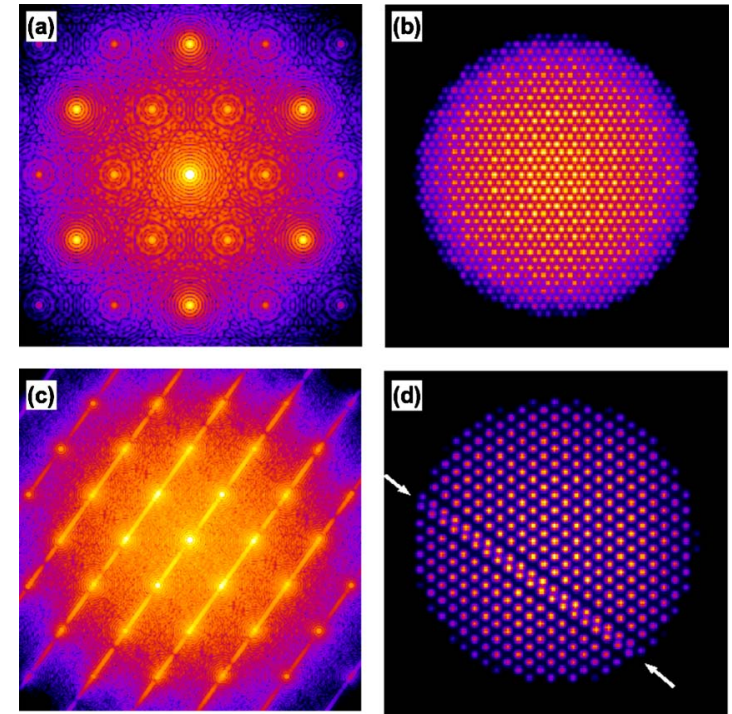

FIG. 5. (Color online) [(a) and (c)] Simulated diffraction patterns of an fcc lattice with the stacking fault defect described in the text. Additional small variations in the positions of the scatterers are introduced. [(b) and (d)] Results of the reconstruction performed as described in the text. [(a) and (b) $] \varphi=0^{\circ},[(\mathrm{c})$ and (d) $] \varphi=35^{\circ}$.

with the same stacking direction. The effect of the stacking fault here is a translation of the two fcc crystals relative to each other. This "sliding" can be seen in Fig. 3(d) as a "break" of the lines of bright spots at the defect. It was recently suggested that, on the basis of $\mathrm{x}$-ray diffraction over a large (submillimeter) sample area, these double stacking defects consisting of two hcp planes is a common imperfection in convectively assembled colloidal crystals. ${ }^{36}$

To support our findings, we present simulations performed on an fcc structure. Our model system was a sphere filled with point scatterers ordered in an fcc lattice. The spherical shape represents the round part of the colloidal sample chosen by the pinhole. All other parameters of the simulation have been chosen to fit with the experiment. We introduced a stacking fault consisting of two hcp planes ${ }^{36}$ as a defect in our model structure. Since our real space reconstruction from the experimental data shows an additional narrowing of two layers [see Fig. 3(d)], we changed the distance between the two adjacent planes surrounding the stacking fault to $2 / 3$ of the nominal (111) distance. To complete the simulations we also introduced a random shift in the positions of the point scatterers. This results in diffraction patterns with poorer contrast than ideal, similar to our measurements. The resulting diffraction patterns, calculated at different orientations of our model sample corresponding to azimuthal angles $\varphi=0^{\circ}$ [Fig. 5(a)] and $\varphi=35^{\circ}$ [Fig. 5(c)], concord very well with our measurements at the same angles [Figs. 2(b) and 2(d)]. They show a strong set of 220 Bragg peaks at $\varphi=0^{\circ}$ orientation [Fig. 5(a)] as well as weak forbidden peaks 1/3(224) due to the presence of the defect in a model sample. At $\varphi=35^{\circ}$ orientation a set of rods connecting the Bragg peaks is clearly observed, similar to our measurements in Fig. 2(d). The inversion of these diffraction patterns [Figs. 5(b) and 5(d)] yield similar results as seen in the reconstruction of the experimental data. 
In Fig. 4(b), a scanning electron microscopy (SEM) image of the crystal surface of a similar colloidal crystal is shown in a [111] view. The morphologies of the SEM image, the reconstructed image in Fig. 3(d) and our model simulations correspond well, indicating that the reconstruction is indeed a 2D projection of a stacking fault measured along the [110] direction. This demonstrates the high sensitivity of the proposed CXDI technique with the possibility of mapping the core of the defect structures in convectively assembled colloidal crystals. For example, coherent imaging can reveal small effects such as the presence of displacements between layers containing a stacking fault [compare Figs. 3(d) and 4(b)]. Note, however, that the SEM image only provides information about the top layer of the crystal, while the CXDI image is a projection through the thickness of the whole $3 \mathrm{D}$ crystal structure.

\section{CONCLUSIONS AND OUTLOOK}

In summary, we have demonstrated that the simple and nondestructive mechanism of coherent $\mathrm{x}$-ray diffractive imaging opens a unique route to determine defects in mesoscopic materials such as colloidal crystals. CXDI together with new hard $\mathrm{x}$-ray microscopy methods ${ }^{37}$ has the potential to provide detailed information about the local defect structure in colloidal crystals. This is especially important for imaging photonic materials when refraction index matching is not possible or the sizes of colloidal particles are too small for conventional optical microscopy. To extend this method to larger fields of view scanning methods such as ptychography ${ }^{38,39}$ can be used, while tomographic methods such as coherent $\mathrm{x}$-ray tomography ${ }^{40}$ have the potential to visualize the atomic structure of the defect core in $3 \mathrm{D}$. Another exciting extension of this method will be its application to imaging protein crystals of a few micrometers size. Revealing the structure and mapping defects in such biological samples could solve important questions about the initial stages of crystal growth of macromolecular assemblies and provide recipes for successful crystallization techniques, which are presently a bottleneck in modern crystallography. Our approach can be conveniently extended for time resolved studies of crystallization and defect formation using the presently emerging $\mathrm{x}$-ray free-electron lasers. ${ }^{41}$

\section{ACKNOWLEDGMENTS}

The authors would like to acknowledge D. Stickler for performing the FIB milling, A. Singer for assistance with the first measurements, R. Roehlsberger for a careful reading of the manuscript and E. Weckert for his interest and support during this project. The authors are grateful to T. Roth and C. Detlefs (ESRF) for their invaluable assistance at the ID06 beamline.

\footnotetext{
*Corresponding author; ivan.vartaniants@desy.de

${ }^{1}$ N. W. Ashcroft and N. Mermin, Solid State Physics (Holt, Rinehart and Winston, New York, 1976).

${ }^{2}$ A. Authier, Dynamical Theory of X-Ray Diffraction (Oxford University Press, New York, 2001).

${ }^{3}$ Advanced Tomographic Methods in Materials Research and Engineering, edited by J. Banhart (Oxford University Press, New York, 2008).

${ }^{4}$ P. Lodahl, A. F. van Driel, I. Nikolaev, A. Irman, K. Overgaag, D. Vanmaekelbergh, and W. L. Vos, Nature (London) 430, 654 (2004).

${ }^{5}$ R. H. T. Bates, Optik (Stuttgart) 61, 247 (1982).

${ }^{6}$ J. Fienup, Appl. Opt. 21, 2758 (1982).

${ }^{7}$ V. Elser, J. Opt. Soc. Am. A 20, 40 (2003).

${ }^{8}$ S. Marchesini, Rev. Sci. Instrum. 78, 011301 (2007).

${ }^{9}$ J. Miao, P. Charalambous, J. Kirz, and D. Sayre, Nature (London) 400, 342 (1999).

${ }^{10}$ K. A. Nugent, Adv. Phys. 59, 1 (2010).

${ }^{11}$ A. P. Mancuso, O. M. Yefanov, and I. A. Vartanyants, J. Biotechnol. (to be published).

${ }^{12}$ I. K. Robinson, I. A. Vartanyants, G. J. Williams, M. A. Pfeifer, and J. A. Pitney, Phys. Rev. Lett. 87, 195505 (2001).

${ }^{13}$ M. A. Pfeifer, G. J. Williams, I. A. Vartanyants, R. Harder, and I. K. Robinson, Nature (London) 442, 63 (2006).

${ }^{14}$ I. Robinson and R. Harder, Nature Mater. 8, 291 (2009).

${ }^{15}$ I. A. Vartanyants and I. K. Robinson, J. Phys.: Condens. Matter 13, 10593 (2001).

${ }^{16}$ J. Gulden, Diploma thesis, University of Rostock, 2008.
}

${ }^{17}$ W. J. Huang, J. M. Zuo, B. Jiang, K. W. Kwon, and M. Shim, Nat. Phys. 5, 129 (2009).

${ }^{18}$ R. Dronyak, K. S. Liang, Y. P. Stetsko, T.-K. Lee, C.-K. Feng, J.-S. Tsai, and F.-R. Chen, Appl. Phys. Lett. 95, 111908 (2009).

${ }^{19}$ A. P. Mancuso, A. Schropp, B. Reime, L.-M. Stadler, A. Singer, J. Gulden, S. Streit-Nierobisch, C. Gutt, G. Grübel, J. Feldhaus, F. Staier, R. Barth, A. Rosenhahn, M. Grunze, T. Nisius, T. Wilhein, D. Stickler, H. Stillrich, R. Frömter, H.-P. Oepen, M. Martins, B. Pfau, C. M. Günther, R. Könnecke, S. Eisebitt, B. Faatz, N. Guerassimova, K. Honkavaara, V. Kocharyan, R. Treusch, E. Saldin, S. Schreiber, E. A. Schneidmiller, M. V. Yurkov, E. Weckert, and I. A. Vartanyants, Phys. Rev. Lett. 102, 035502 (2009).

${ }^{20}$ A. V. Petukhov, I. P. Dolbnya, D. G. A. L. Aarts, G. J. Vroege, and H. N. W. Lekkerkerker, Phys. Rev. Lett. 90, 028304 (2003).

${ }^{21}$ P. Jiang, J. F. Bertone, K. S. Hwang, and V. L. Colvin, Chem. Mater. 11, 2132 (1999).

${ }^{22}$ A. Blanco, E. Chomski, S. Grabtchak, M. Ibisate, S. John, S. W. Leonard, C. Lopez, F. Meseguer, H. Miguez, J. P. Mondia, G. A. Ozin, O. Toader, and H. M. van Driel, Nature (London) 405, 437 (2000).

${ }^{23}$ J. W. Goodwin, J. Hearn, C. C. Ho, and R. H. Ottewill, Colloid Polym. Sci. 252, 464 (1974)

${ }^{24}$ J. Hilhorst, V. V. Abramova, A. Sinitskii, N. A. Sapoletova, K. S. Napolskii, A. A. Eliseev, D. V. Byelov, N. A. Grigoryeva, A. V. Vasilieva, W. G. Bouwman, K. Kvashnina, A. Snigirev, S. V. Grigoriev, and A. V. Petukhov, Langmuir 25, 10408 (2009).

${ }^{25} \mathrm{M}$. Born and E. Wolf, Principles of Optics, 6th ed. (Cambridge 
University Press, Cambridge, England, 2000).

${ }^{26}$ C.-C. Chen, J. Miao, C. W. Wang, and T. K. Lee, Phys. Rev. B 76, 064113 (2007).

${ }^{27}$ P. Thibault, V. Elser, C. Jacobsen, D. Shapiro, and D. Sayre, Acta Crystallogr. A 62, 248 (2006).

${ }^{28}$ I. A. Vartanyants and I. K. Robinson, J. Synchrotron Radiat. 10, 409 (2003).

${ }^{29}$ B. E. Warren, X-Ray Diffraction (Dover Publications Inc., New York, 1990).

${ }^{30}$ P. N. Pusey, W. van Megen, P. Bartlett, B. J. Ackerson, J. G. Rarity, and S. M. Underwood, Phys. Rev. Lett. 63, 2753 (1989).

${ }^{31}$ W. Loose and B. J. Ackerson, J. Chem. Phys. 101, 7211 (1994).

${ }^{32}$ H. Versmold, Phys. Rev. Lett. 75, 763 (1995).

${ }^{33}$ R. M. Amos, J. G. Rarity, P. R. Tapster, T. J. Shepherd, and S. C. Kitson, Phys. Rev. E 61, 2929 (2000).

${ }^{34}$ H. J. Schöpe, A. B. Fontecha, H. König, J. M. Hueso, and R. Biehl, Langmuir 22, 1828 (2006).

${ }^{35}$ I. P. Dolbnya, A. V. Petukhov, D. G. A. L. Aarts, G. J. Vroege, and H. N. W. Lekkerkerker, EPL 72, 962 (2005).

${ }^{36}$ K. S. Napolskii, N. A. Sapoletova, D. F. Gorozhankin, A. A.
Eliseev, D. Y. Chernyshov, D. V. Byelov, N. A. Grigoryeva, A. A. Mistonov, W. G. Bouwman, K. O. Kvashnina, A. V. Lukashin, A. A. Snigirev, A. V. Vassilieva, S. V. Grigoriev, and A. V. Petukhov, Langmuir 26, 2346 (2010).

${ }^{37}$ A. Bosak, I. Snigireva, K. S. Napolskiia, and A. Snigirev, Adv. Mater. (to be published).

${ }^{38}$ J. M. Rodenburg, A. C. Hurst, A. G. Cullis, B. R. Dobson, F. Pfeiffer, O. Bunk, C. David, K. Jefimovs, and I. Johnson, Phys. Rev. Lett. 98, 034801 (2007).

${ }^{39}$ P. Thibault, M. Dierolf, A. Menzel, O. Bunk, C. David, and F. Pfeiffer, Science 321, 379 (2008).

${ }^{40}$ O. M. Yefanov, A. V. Zozulya, I. A. Vartanyants, J. Stangl, C. Mocuta, T. H. Metzger, G. Bauer, T. Boeck, and M. Schmidbauer, Appl. Phys. Lett. 94, 123104 (2009).

${ }^{41}$ I. A. Vartanyants, I. K. Robinson, I. McNulty, C. David, P. Wochner, and T. Tschentscher, J. Synchrotron Radiat. 14, 453 (2007).

${ }^{42}$ We neglect here a small contribution of the forbidden $1 / 3$ [224] reflections. 